\title{
Concomitant bilateral medial malleolus stress fracture: a case report and review of literature of an extremely rare injury
}

\author{
Vivek Mathew Philip ${ }^{1 *}$, Raj Shekhar', Saurabh Sharma², Prashant Padhi ${ }^{3}$
}

\begin{abstract}
${ }^{1}$ Department of Orthopedics, Military Hospital, Secunderabad, Hyderabad, Telangana, India
${ }^{2}$ Department of Orthopedics, Army Hospital (R and R), Delhi Cantt, New Delhi, India

${ }^{3}$ Department of Orthopedics, 7 Air Force Hospital, Kanpur, Uttar Pradesh, India
\end{abstract}

Received: 29 December 2021

Accepted: 07 February 2022

\author{
*Correspondence: \\ Dr. Vivek Mathew Philip, \\ E-mail: drvivekortho121@gmail.com
}

Copyright: () the author(s), publisher and licensee Medip Academy. This is an open-access article distributed under the terms of the Creative Commons Attribution Non-Commercial License, which permits unrestricted non-commercial use, distribution, and reproduction in any medium, provided the original work is properly cited.

\begin{abstract}
Medial malleolus stress fractures are rare stress injuries and account for $4 \%$ of all the stress fractures. Bilateral medial malleolus stress fracture (MMSFs) is extremely rare with only five such cases reported in existing literature. We report an extremely rare injury pattern in the form of concomitant bilateral MMSF in a young military recruit who presented with severe pain in both his ankles for one month. A 20-year military recruit undergoing rigorous physical training presented to us with severe bilateral ankle pain of one month duration. Clinico-radiological examination revealed bilateral MMSFs. He was managed with open reduction and internal fixation using a one-third tubular plate in buttress mode. Patient was gradually rehabilitated and on one-year follow up, he is asymptomatic and performing all strenuous physical activities. Bilateral MMSFs are extremely rare injuries with only five such cases reported in existing medical literature and this is the fourth instance where bilateral MMSFs were diagnosed at the time of clinical presentation and managed simultaneously, in the same surgical setting.
\end{abstract}

Keywords: Stress fracture, Medial malleolus, Bilateral, Concomitant

\section{INTRODUCTION}

Medial malleolus stress fractures (MMSFs) constitute only less than $5 \%$ of stress fractures. ${ }^{1}$ It is most commonly diagnosed in young individuals involved in strenuous physical activities like military recruits, track athletes and basketball players. ${ }^{2-4}$ The diagnosis is usually made with the complaint of activity related pain, tenderness over the medial malleolus and the radiological finding of a vertical fracture line at the level of the medial corner of tibial plafond. ${ }^{5}$ Bilateral MMSFs are exceedingly rare with only three such instances reported in the existing medical literature. ${ }^{6-8}$ The management of MMSFs is preferably surgical due to higher chances of complications viz, delayed union, non-union and fracture propagation. ${ }^{3,4,9}$ The case reported is the second instance ever with simultaneous presentation of MMSF of both ankles in young individuals ; managed surgically in the same setting with excellent postoperative recovery.

\section{CASE REPORT}

A 20-year-old military recruit presented with the complaints of gradually worsening activity related pain on the inner side of both ankles of one month duration. Clinical examination on presentation revealed bilateral ankle effusion and tenderness over both medial malleoli. There was no limb shortening or angular deformity of both lower limbs. Plain radiographs revealed a vertically oriented fracture line involving the medial border of the tibial plafond on both sides (Figure 1). A metabolic work up was done to look for any evidence of nutritional/metabolic insufficiency. The metabolic panel was normal with a serum calcium level of $9.5 \mathrm{mg} / \mathrm{dl}(8.5$ $10.5 \mathrm{mg} / \mathrm{dl})$, vitamin D 25-OH level of $42 \mathrm{ng} / \mathrm{ml}(20-150$ $\mathrm{ng} / \mathrm{ml})$, serum alkaline phosphatase level of $185 \mathrm{~mol} / \mathrm{L}$ (65-260 mol/L), serum parathormone levels of $21 \mathrm{pg} / \mathrm{ml}$ $(10-55 \mathrm{pg} / \mathrm{ml})$. Due to higher chances of previously mentioned complications of conservative management, the 
patient underwent surgical management of both MMSF in the same surgical setting. Under spinal anaesthesia block, patient underwent open reduction and buttress plating using stainless steel one-third tubular plates on both limbs (Figure 2). Post-operatively, the patient was encouraged to perform active ankle range of movements immediately, protected weight bearing was started after four weeks, full weight bearing started after six weeks, although he was excused from strenuous physical activities like running and jumping for six months. On one year follow-up, the patient had full clinical and radiological union of his fractures and was performing all is training activities without any symptoms (Figure 3).

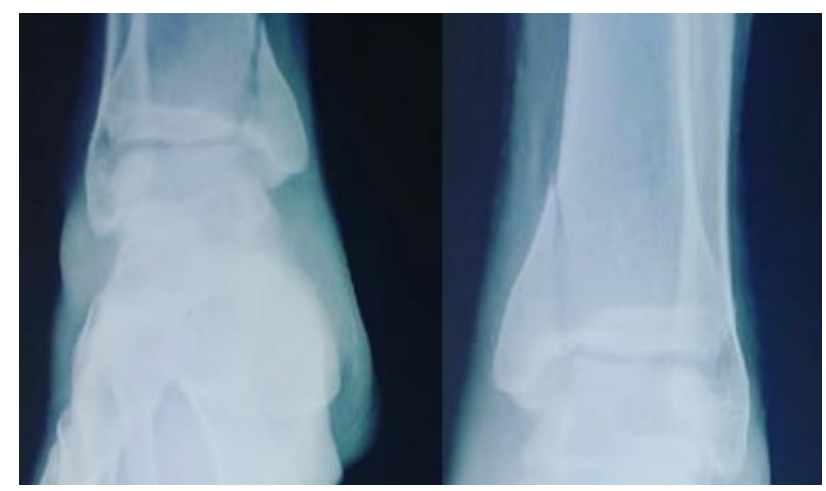

Figure 1: Pre-operative X-ray demonstrating bilateral MMSF.

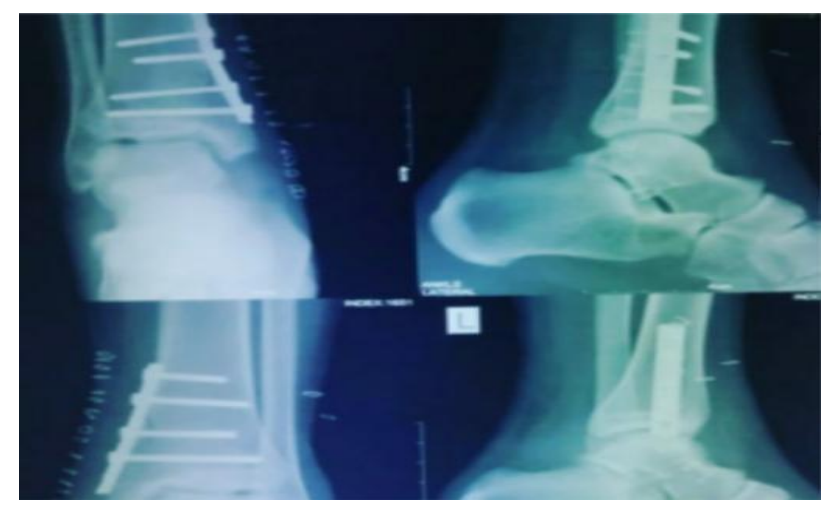

Figure 2: Immediate postoperative $\mathrm{X}$-ray after osteosynthesis.

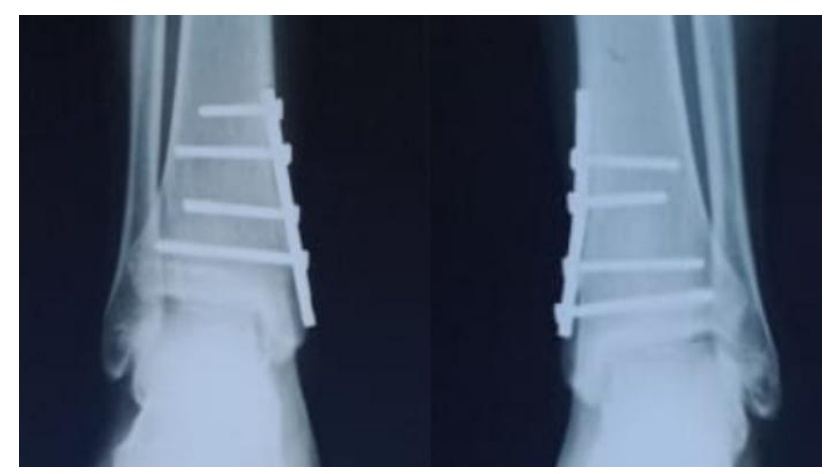

Figure 3: X-ray at one year follow-up showing complete radiological union.

\section{DISCUSSION}

Stress fractures occur due to repetitive abnormal mechanical loading of normal bone. ${ }^{10}$ The incidence of stress fractures of the medial malleolus varies from 0.6 to $4.1 \%$ of all stress fractures and is described most commonly in young skeletally mature athletes involved in strenuous physical activities like running and jumping. Risk factors for stress fractures can be broadly divided into three groups: activity related, which include poor footwear, excessive training and irregular terrain; biomechanical factors including inflexible or weak calf muscles, unequal leg length, flat or high-arched feet, forefoot varus, subtalar varus, and tibia vara; and metabolic factors like hormonal imbalance, osteoporosis, renal osteodystrophy, nutritional deficiencies, rheumatoid arthritis and osteoarthritis. ${ }^{11}$ Bilateral MMSF are extremely rare injuries reported only a few times in existing medical literature. AP plain radiograph of the ankle is the initial investigation of choice for diagnosing MMSF. Negative radiographs with high clinical suspicion should be further evaluated with MRI or CT imaging for diagnosis. ${ }^{5}$ Steckel et al reported the occurrence of bilateral MMSF in a young athlete, but the presentations were sequential over a period of 21 months and was managed surgically to return to full function. ${ }^{6}$ Looze et al reported a case of bilateral MMSF in a 60-year-old post-menopausal woman, which was described as more of an "insufficiency" fracture rather than a stress fracture. The patient underwent surgical management and achieved her baseline functional status at one year follow-up. ${ }^{12}$ Soni et al reported a case of bilateral MMSF in a 65-year-old lady secondary to osteoarthritis of both knees. They managed the case surgically with osteosynthesis on both sides and the patient subsequently underwent total knee arthroplasty for osteoarthritis of both knees. Patient was asymptomatic at six months of follow up. ${ }^{11}$ Kanto et al managed a case of bilateral MMSF in a young athlete, sequentially over a period of two months with osteosynthesis prompting an excellent post-operative recovery. ${ }^{7}$ Klavas et al described a case of bilateral MMSF in a sixteen year old high school athlete who was managed surgically on both sides simultaneously in the same surgical setting with excellent result at six month follow up. ${ }^{8}$ In the present case, the patient suffered bilateral MMSF after one month of intense physical excretion. Clinical examination showed no limb deformities or limb length discrepancy. The lab parameters were normal, ruling out metabolic/ hormonal insufficiencies. Like previously reported cases, the patient underwent surgical fixation to preclude the chances of delayed union or non-union and due to the involvement of the weight bearing tibial plafond. Simultaneous osteosynthesis using one-third tubular plates applied in a buttress mode ensured adequate fracture reduction and immediate commencement of ankle range of motion. Patient was not allowed to bear weight for a short period of four weeks and subsequently allowed weight bearing according to tolerance. At one year of follow up, the patient was asymptomatic and had features of clinical and radiological fracture healing. 


\section{CONCLUSION}

The clinical significance of this case report is that prompt diagnosis of bilateral MMSF, surgical management and mitigation of the possible risk factors mentioned above will help the patients in regaining their pre-injury level of physical activity. It would be prudent for patients presenting with activity associated ankle pain with normal findings on plain radiographs to undergo further imaging in the form of CT scan or MRI to rule out an occult stress fracture. A medial malleolus stress fracture is a rare but well-documented injury in running and jumping athletes. Bilateral MMSF are extremely rare and have previously been reported in five instances as case reports. In this case, prompt diagnosis, a relatively simple surgical fixation and focussed post operative rehabilitation ensured clinical and radiological healing of the fractures and return to preinjury level of physical activity at 01 year of follow up.

Funding: No funding sources

Conflict of interest: None declared

Ethical approval: Not required

\section{REFERENCES}

1. Nguyen A, Beasley I, Calder J. Stress fractures of the medial malleolus in the professional soccer player demonstrate excellent outcomes when treated with open reduction internal fixation and arthroscopic spur debridement. Knee Surg Sports Traumatol Arthrosc. 2019;27:2884-9.

2. Orava S, Karpakka J, Taimela S, Hulkko A, Permi J, Kujala U. Stress fracture of the medial malleolus. J Bone Joint Surg Am. 1995;77:362-5.

3. Anderson RB, Hunt KJ, McCormick JJ: Management of common sports-related injuries about the foot and ankle. J Am Acad Orthop Surg. 2010;18:546-56.
4. Irion V, Miller TL, Kaeding CC. The treatment and outcomes of medial malleolar stress fractures: a systematic review of the literature. Sports Health. 2014;6:527-30.

5. Lempainen L, Liimatainen E, Heikkilä J, Alonso J, Sarimo J, Mattila K et al. Medial malleolar stress fracture in athletes: diagnosis and operative treatment. Scand J Surg. 2012;101:261-4.

6. Steckel H, Klinger HM, Baums MH, Schultz W. Beidseitige stressfraktur des malleolus medialis," Sportverletz Sportschaden. 2005;19(1):41-5.

7. Kanto R, Fukunishi S, Marooka T, Seino D, Takashima T, Yoshiya A et al. Double threaded screw fixation for bilateral stress fracture of the medial malleolus. Case Rep Orthop. 2014;729035.

8. Klavas DM, Holderread BM, Liu J. Bilateral Medial Malleolus Stress Fractures in a High School Athlete Treated with Open Reduction Internal Fixation. Cureus. 2021;13(9):e18186.

9. Reider B, Falconiero R, Yurkofsky J. Non-union of a medial malleolus stress fracture. A Case Report. Am J Sports Med. 1993;21:478-81.

10. Boden BP, Osbahr DC. High-risk stress fractures: evaluation and treatment. J Am Acad Orthop Surg. 2000;8:344-53.

11. Ashish S, Samuel V, Kurt H, Aaron N. Bilateral medial malleolus stress fractures due to osteoarthritis of knee: A case report and review of literature. Int $\mathbf{J}$ Surg Case Rep. 201;6C:2014;266-8.

12. Looze CA, BS, Golden B, MD, Egol KA. Bilateral Fractures of the Medial Malleoli Without a History of Trauma.Am J Orthop. 2009;38(5):237-40.

Cite this article as: Philip VM, Shekhar R, Sharma $\mathrm{S}$, Padhi P. Concomitant bilateral medial malleolus stress fracture: a case report and review of literature of an extremely rare injury. Int J Res Orthop 2022;8:275-7. 